\title{
A Mixed Formulation of Stabilized Nonconforming Finite Element Method for Linear Elasticity
}

\author{
Bei Zhang ${ }^{1}$ and Jikun Zhao ${ }^{2, *}$ \\ ${ }^{1}$ College of Science, Henan University of Technology, Zhengzhou, Henan 450001, China \\ 2 School of Mathematics and Statistics, Zhengzhou University, Zhengzhou, Henan 450001, \\ China
}

Received 22 February 2019; Accepted (in revised version) 5 August 2019

\begin{abstract}
Based on the primal mixed variational formulation, a stabilized nonconforming mixed finite element method is proposed for the linear elasticity problem by adding the jump penalty term for the displacement. Here we use the piecewise constant space for stress and the Crouzeix-Raviart element space for displacement. The mixed method is locking-free, i.e., the convergence does not deteriorate in the nearly incompressible or incompressible case. The optimal convergence order is shown in the $L^{2}$-norm for stress and in the broken $H^{1}$-norm and $L^{2}$-norm for displacement, respectively. Finally, some numerical results are given to demonstrate the optimal convergence and stability of the mixed method.
\end{abstract}

AMS subject classifications: 65N15, 65N30

Key words: Mixed method, nonconforming finite element, elasticity, locking-free, stabilization.

\section{Introduction}

Different variational formulations of the linear elasticity problem have been developed in the past. In the sense of weak form, it is possible to show the equivalence to the classical formulation, which consists of the constitutive equation and the equilibrium equation and is well-defined for differentiable stress and displacement. A very common formulation is the pure displacement formulation, where only the displacement is sought in $H^{1}$-space. Therein, the stress tensor is eliminated by using the constitutive equation, as it can be expressed in terms of the displacement for finite $\lambda$ where $\lambda$ is the Lamé constant. However, when this formulation is used, one may expect the stability problem in case of nearly incompressible or incompressible materials, such that the locking phenomenon may appear. This is due to the fact that, the compliance tensor, which links

*Corresponding author.

Emails: beizhang@haut.edu.cn (B. Zhang), jkzhao@zzu.edu.cn (J. K. Zhao) 
the stress and strain, becomes singular in the incompressible limit (the case $\lambda=\infty$ ), such that the elasticity tensor, the inverse of the compliance tensor acting as a coefficient in the pure displacement formulation, deteriorates with the growing incompressibility of material $(\lambda \rightarrow \infty)$. In the incompressible limit, the inverse of compliance tensor doesn't exist, so the stress can not be expressed in terms of the displacement, such that it is not possible to pose the pure displacement formulation in this case. For this formulation, some locking-free finite element methods have been developed where the convergence is uniform with respect to the Lamé constant $\lambda$, see, e.g., $[9,13,21,30,31,40]$, which will be further discussed in the following.

Relatively, the mixed variational formulations are preferable to the pure displacement formulation for modeling of nearly incompressible or incompressible materials, where both the stress and displacement are simultaneously considered as unknowns as they show better stability properties. Moreover, the stress is usually a physical quantity of primary interest. Although it can be obtained in the pure displacement method by differentiating the displacement, but this may degrade the order of the approximation.

Here we consider the Hellinger-Reissner mixed formulation. There are essentially two possibilities to apply the derivatives to the displacement or the stress. The first one is the primal mixed variational formulation, which is easy to discretize but leads usually to the standard methods suffering from locking unless special techniques are applied. Before surveying further the first one, we browse the second one called the dual mixed variational formulation. The second one needs stress tensor elements with continuous normal components, which is very difficult to construct due to the symmetry and stability requirements from this formulation, but may lead to better approximation properties. While such stable conforming element pairs have been successfully constructed in both two and three dimensions, the resulting stress elements tend to be quite complicated, especially in three dimensions, see e.g., $[1,2,5,6,27]$. We also mention the further development of stable conforming elements from the references $[12,22,24,25]$, where a new class of stable conforming elements called the Hu-Zhang element is proposed. On the other hand, much attention has been paid to constructing the nonconforming mixed finite elements, which relax the interelement continuity requirement and seem to be simpler, see $[3,7,23]$ and the references therein. However, the convergence of nonconforming mixed finite element methods has not been considered for nearly incompressible or incompressible materials in the works mentioned above, where the compliance tensor is only assumed to be bounded and symmetric positive definite. Besides, we mention that, some mixed elements with weaker symmetry have been developed and can be shown to be uniformly convergent with respect to the Lamé constant $\lambda$, see, e.g., $[4,29,32,35]$.

Now we turn back to the primal mixed variational formulation. As mentioned before, for finite $\lambda$ this formulation can be reduced to the pure displacement formulation by eliminating the stress. By splitting the elasticity operator into the gradient operator and divergence operator with appropriate coefficients, Brenner and Sung [9] used the Crouzeix-Raviart (CR) element [17] to develop a locking-free nonconforming finite element method for the pure displacement problem of nearly incompressible elasticity. 
Similar ideas can be found in $[13,31]$. However, in the case of the elasticity operator, the $\mathrm{CR}$ element is not stable in that it does not fulfill a discrete Korn's inequality. For this reason, Hansbo and Larson [21] proposed a stabilized nonconforming method for the CR element by adding a jump penalty term based on the pure displacement formulation, where the optimal convergence order was shown uniformly in the nearly incompressible case. Therein, the error analysis was carried out in a mesh dependent energy-like norm (see Section 7 for the definition). There also exist some other numerical approaches to deal with the locking phenomenon. For example, one may resort to the reduced integration or some modifications of the variational formulation. Many works are involved in them directly or indirectly. The readers are referred to $[18,28,30,40]$ for the detailed analysis. An alternative way to avoid locking is to use high-order elements as is done in $[36,37]$ and leads to the $p$-version and $h p$-version methods. By contrast, the mixed finite element method based on the primal mixed variational formulation seems to be more effective for nearly incompressible or incompressible materials than the pure displacement methods and the corresponding discrete spaces are easier to constructed than the mixed methods based on the dual mixed variational formulation, see [19] for the work on this aspect. For further developments, see [11,34,39].

In this paper, we focus on the discretization of the primal mixed variational formulation. A locking-free stabilized nonconforming mixed finite element method is proposed based on the primal mixed variational formulation by adding the jump penalty term for the displacement, where we use the piecewise constant space for stress and the CR element space for displacement. We derive a weaker inf-sup condition on a special subspace of the discrete space, which is enough to show the optimal convergence properties of the mixed method. Based on this, the convergence order is shown to be $\mathcal{O}(h)$ in the $L^{2}$-norm for stress and in the broken $H^{1}$-norm for displacement. By using the usual duality argument, the convergence order is shown to be $\mathcal{O}\left(h^{2}\right)$ in the $L^{2}$-norm for displacement. It is worth mentioning that, for finite $\lambda$ our mixed method can be reduced to the stabilized nonconforming method [21] based on the pure displacement formulation, where the error analysis was carried out in a mesh dependent energy-like norm as mentioned above. By contrast, the norms used in this paper are more natural and completely independent of the mesh size and the Lamé constant, see Section 7 for the discussion on this.

\section{Linear elasticity}

Let $\Omega$ be a bounded domain in $\mathbb{R}^{d}$ with the dimension $d=2,3$. For any given open subset $S$ of $\Omega,(\cdot, \cdot)_{S}$ and $\|\cdot\|_{S}$ denote the usual integral inner product and the corresponding norm of $L^{2}(S)^{d}$, respectively. For a positive integer $m$, we shall use the common notations for the Sobolev spaces $H^{m}(S)$ and $H_{0}^{m}(S)$ with the corresponding norms $\|\cdot\|_{m, S}$ and $|\cdot|_{m, S}$ (see e.g., [15]). If $S=\Omega$, the subscript will be omitted. We use $H^{-1}(\Omega)$ to denote the dual space of $H_{0}^{1}(\Omega)$ with respect to the duality product $\langle\cdot, \cdot\rangle$. The dual norm in $H^{-1}(\Omega)$ is 
defined by

$$
\|v\|_{-1}=\sup _{\phi \in H_{0}^{1}(\Omega) \backslash\{0\}} \frac{\langle v, \phi\rangle}{\|\phi\|_{1}}, \quad v \in H^{-1}(\Omega) .
$$

Let $\sigma$ and $u$ be the symmetric stress tensor and the displacement field. Denote $\varepsilon(u)=$ $\left(\varepsilon_{i j}(u)\right)_{d \times d}$ as the linearized strain tensor, where

$$
\varepsilon_{i j}(u)=\frac{1}{2}\left(\frac{\partial u_{i}}{\partial x_{j}}+\frac{\partial u_{j}}{\partial x_{i}}\right)
$$

The relation between the stress and the strain tensors can be expressed as the constitutive law:

$$
\sigma=\mathcal{C} \varepsilon(u) \quad \text { or } \quad \varepsilon(u)=\mathcal{A} \sigma,
$$

where $\mathcal{C}$ and $\mathcal{A}$ are the elasticity and the compliance tensors of fourth order, respectively. For an isotropic elastic material, they have the following expressions:

$$
\begin{aligned}
& \mathcal{C} \varepsilon(u)=2 \mu \varepsilon(u)+\lambda(\operatorname{tr} \varepsilon(u)) \delta, \\
& \mathcal{A} \sigma=\frac{1}{2 \mu}\left(\sigma-\frac{\lambda}{d \lambda+2 \mu} \operatorname{tr} \sigma \delta\right),
\end{aligned}
$$

respectively. Here $\delta=\left(\delta_{i j}\right)_{d \times d}$ is the identity tensor, $\lambda$ and $\mu$ are the Lamé constants such that $\mu \in\left[\mu_{1}, \mu_{2}\right]$ with $0<\mu_{1}<\mu_{2}$ and $\lambda \in(0, \infty]$. When $\lambda$ is very large or infinite, materials are said to be nearly incompressible or incompressible.

For finite $\lambda$, it is clear that $\mathcal{C}$ is symmetric and positive definite and further $\mathcal{A}=\mathcal{C}^{-1}$. When $\lambda$ approaches infinity, the elasticity tensor blows up and the compliance tensor tends to $\frac{1}{2 \mu} \mathrm{dev}$ that is not invertible, where $\operatorname{dev} \sigma$ is the deviatoric part of $\sigma$, i.e., $\operatorname{dev} \sigma=$ $\sigma-\frac{1}{d} \operatorname{tr} \sigma \delta$. Hence, for nearly incompressible or incompressible materials, it is preferable to use the following relation between the stress and the strain:

$$
\mathcal{A} \sigma=\varepsilon(u) .
$$

In view of the above discussion, we consider the linear elasticity problem: find the stress tensor $\sigma$ and the displacement field $u$ satisfying

$$
\begin{cases}\mathcal{A} \sigma-\varepsilon(u)=0 & \text { in } \Omega, \\ -\operatorname{div} \sigma=f & \text { in } \Omega, \\ u=0 & \text { on } \partial \Omega,\end{cases}
$$

where $f \in H^{-1}(\Omega)^{d}$ is the body force. For simplicity, here we assume that the boundary condition is homogeneous. If the domain $\Omega$ is convex or its boundary is enough smooth, the regularity estimate

$$
\|\sigma\|_{1}+\|u\|_{2} \leq C\|f\|
$$


holds, cf. $[5,9,16,20,33]$. Henceforth, the symbol $C$ with or without subscripts is used to denote a generic positive constant, possibly different at different occurrences, which is independent of the Lamé constant $\lambda$ and the mesh size. For clearness, we also use other symbols (e.g., $\alpha, \beta, \gamma$ ) to denote such a generic positive constant.

\section{Primal mixed variational formulation}

Before displaying the mixed variational formulation of elasticity problem (2.2), let us first describe the solution space. Since $u$ vanishes on $\partial \Omega$, we have

$$
\int_{\Omega} \operatorname{div} u \mathrm{~d} x=\int_{\partial \Omega} u \cdot n \mathrm{~d} s=0,
$$

which leads to

$$
\int_{\Omega} \operatorname{tr} \sigma \mathrm{d} x=0
$$

Therefore, we can impose such a condition on the stress $\sigma$. Define the spaces

$$
\Sigma=\left\{\tau \in L^{2}(\Omega, S) ; \int_{\Omega} \operatorname{tr} \tau \mathrm{d} s=0\right\}, \quad V=H_{0}^{1}(\Omega)^{d},
$$

where $L^{2}(\Omega, S)$ stands for the space of symmetric tensors in $L^{2}(\Omega)^{d \times d}$.

Then the primal mixed variational formulation of (2.2) reads: find $(\sigma, u) \in \Sigma \times V$ satisfying

$$
\begin{cases}(\mathcal{A} \sigma, \tau)-(\tau, \varepsilon(u))=0, & \forall \tau \in \Sigma \\ (\sigma, \varepsilon(v))=(f, v), & \forall v \in V\end{cases}
$$

which is equivalent to the standard $H^{1}$-based variational formulation (pure displacement formulation). In order to obtain the existence and uniqueness of the solution to (3.1), the following two stability conditions is well-known:

Z-ellipticity. There exists a positive constant $\alpha$ such that

$$
\alpha\|\tau\|^{2} \leq(\mathcal{A} \tau, \tau), \quad \forall \tau \in Z,
$$

where $Z=\{\tau \in \Sigma ;(\tau, \varepsilon(v))=0, \forall v \in V\}$.

Inf-sup condition. There exists a positive constant $\beta$ such that, for any $v \in V$,

$$
\sup _{\tau \in \Sigma \backslash\{0\}} \frac{(\tau, \varepsilon(v))}{\|\tau\|} \geq \beta|v|_{1} .
$$

To conclude the section, we introduce a fundamental inequality on the trace of $\Sigma$ :

$$
\|\operatorname{tr} \tau\|^{2} \leq C\left((\mathcal{A} \tau, \tau)+\|\operatorname{div} \tau\|_{-1}^{2}\right), \quad \forall \tau \in \Sigma .
$$


This inequality has been proven for a subspace consisting of the tensors in $\Sigma$ with squareintegrable weak divergences, see [5] for the two dimensional case or also [10] for the general case. In fact, it also holds for the whole space $\Sigma$ and the proof only needs some obvious modifications based on the original one.

According to the definition $(2.1 \mathrm{~b})$ of $\mathcal{A}$, we have

$$
(\mathcal{A} \tau, \tau)=\frac{1}{2 \mu}\left(\|\tau\|^{2}-\frac{\lambda}{d \lambda+2 \mu}\|\operatorname{tr} \tau\|^{2}\right)
$$

which, together with (3.4), leads to

$$
\alpha\|\tau\|^{2} \leq(\mathcal{A} \tau, \tau)+\|\operatorname{div} \tau\|_{-1}^{2}, \quad \forall \tau \in \Sigma .
$$

\section{Nonconforming mixed finite element method}

In order to define the finite element spaces, we introduce a family of shape-regular simplicial meshes $\left\{\mathcal{T}_{h}\right\}$ of $\Omega$. For a given mesh $\mathcal{T}_{h}$, we denote the set of all the edges (faces) in $\mathcal{T}_{h}$ by $\mathcal{E}_{h}$ and the set of interior by $\mathcal{E}_{h}^{\text {int }}$, respectively. For an element $K \in \mathcal{T}_{h}$ and an edge (or face) $E \in \mathcal{E}_{h}$, let $h_{K}$ be the diameter of $K$ and $h_{E}$ the diameter of $E$. Especially, we set $h=\max _{K \in \mathcal{T}_{h}}\left\{h_{K}\right\} . n_{K}$ always denotes the exterior unit normal vector along the boundary of $K$. For each edge (face), we define its unit normal vector denoted by $n$, whose orientation is chosen arbitrarily but fixed for interior edges (faces) and coinciding with the exterior normal of $\Omega$ for boundary edges (faces). For a function $v$ and an interior edge (face) $E$ shared by the elements $K$ and $L$ in $\mathcal{T}_{h}$ such that $\left.n\right|_{E}$ points from $K$ to $L$, we define the jump operator $[[\cdot]]$ through $E$ by

$$
\left.[[v]]\right|_{E}=\left.\left(\left.v\right|_{K}\right)\right|_{E}-\left.\left(\left.v\right|_{L}\right)\right|_{E} .
$$

For any boundary edge (face) $E$, set $\left.[[v]]\right|_{E}=\left.v\right|_{E}$. Similar notation is used for the jump of vector-valued functions, where the jump is taken componentwise.

For any given element $K$ and nonnegative integer $m, \mathbb{P}_{m}(K)$ denotes the space of polynomials of order $m$ or less and $\mathbb{P}_{m}(K, \mathbb{S})$ the space of symmetric tensors in $\mathbb{P}_{m}(K)^{d \times d}$.

On $\mathcal{T}_{h}$, we introduce a pair of finite element spaces

$$
\begin{aligned}
& \Sigma_{h}=\left\{\tau_{h} \in \Sigma ;\left.\tau_{h}\right|_{K} \in \mathbb{P}_{0}(K, S), \forall K \in \mathcal{T}_{h}\right\}, \\
& V_{h}=\left\{v_{h} \in L^{2}(\Omega)^{d} ;\left.v_{h}\right|_{K} \in \mathbb{P}_{1}(K)^{d}, \forall K \in \mathcal{T}_{h} \text { and } \int_{E}\left[\left[v_{h}\right]\right] \mathrm{d} s=0, \forall E \in \mathcal{E}_{h}\right\},
\end{aligned}
$$

where $V_{h}$ is the well-known Crouzeix-Raviart finite element space [17].

As usual, we define the interpolation operators $\Pi_{h}$ and $I_{h}$ for the spaces $\Sigma_{h}$ and $V_{h}$ by setting

$$
\begin{array}{ll}
\int_{K} \Pi_{h} \tau \mathrm{d} x=\int_{K} \tau \mathrm{d} x, & \forall K \in \mathcal{T}_{h}, \\
\int_{E} I_{h} v \mathrm{~d} s=\int_{E} v \mathrm{~d} s, & \forall E \in \mathcal{E}_{h},
\end{array}
$$


where $\tau \in L^{2}(\Omega, S)$ and $v \in H^{1}(\Omega)^{d}$. For $\tau \in \Sigma$ and $v \in V$, it obviously holds that $\Pi_{h} \tau \in \Sigma_{h}$ and $I_{h} v \in V_{h}$. Furthermore, by the standard scaling arguments we have the following local interpolation error estimates:

$$
\begin{array}{ll}
\left\|\tau-\Pi_{h} \tau\right\|_{K} \leq C h_{K}^{l}|\tau|_{l, K,} & l=0,1, \\
\left|v-I_{h} v\right|_{m, K} \leq C h_{K}^{l-m}|v|_{l, K}, & m=0,1, \quad l=1,2 .
\end{array}
$$

If we directly discretize the primal mixed variational problem (3.1) with the finite dimensional spaces defined above, it leads to an unstable formulation:

$$
\begin{cases}\left(\mathcal{A} \sigma_{h}, \tau_{h}\right)-\left(\tau_{h}, \varepsilon_{h}\left(u_{h}\right)\right)=0, & \forall \tau_{h} \in \Sigma_{h} \\ \left(\sigma_{h}, \varepsilon_{h}\left(v_{h}\right)\right)=\left(f, v_{h}\right), & \forall v_{h} \in V_{h}\end{cases}
$$

where $\varepsilon_{h}\left(v_{h}\right)$ is defined locally on each $K \in \mathcal{T}_{h}$, i.e., $\left.\varepsilon_{h}\left(v_{h}\right)\right|_{K}=\varepsilon\left(\left.v_{h}\right|_{K}\right)$. In fact, we can only verify the following two conditions:

$Z_{h}$-ellipticity. There exists a positive constant $\alpha$ that is the same as the one in (3.2) such that

$$
\alpha\left\|\tau_{h}\right\|^{2} \leq\left(\mathcal{A} \tau_{h}, \tau_{h}\right), \quad \forall \tau_{h} \in Z_{h}
$$

where $Z_{h}=\left\{\tau_{h} \in \Sigma_{h} ;\left(\tau_{h}, \varepsilon_{h}\left(v_{h}\right)\right)=0, \forall v_{h} \in V_{h}\right\}$.

Weaker inf-sup condition. There exist two positive constants $\beta_{1}$ and $\beta_{2}$ such that, for any $v_{h} \in V_{h}$

$$
\sup _{\tau_{h} \in \Sigma_{h} \backslash\{0\}} \frac{\left(\tau_{h}, \varepsilon_{h}\left(v_{h}\right)\right)}{\left\|\tau_{h}\right\|} \geq \beta_{1}\left\|v_{h}\right\|_{1, h}-\beta_{2}\left(\sum_{E \in \mathcal{E}_{h}} h_{E}^{-1}\left\|\left[\left[v_{h}\right]\right]\right\|_{E}^{2}\right)^{\frac{1}{2}},
$$

where $\|\cdot\|_{1, h}$ is the broken $H^{1}$-norm on the discrete space $V_{h}$ defined by

$$
\left\|v_{h}\right\|_{1, h}=\left(\sum_{K \in \mathcal{T}_{h}}\left|v_{h}\right|_{1, K}^{2}\right)^{\frac{1}{2}} .
$$

To verify the $Z_{h}$-ellipticity, we first give the following lemma.

Lemma 4.1. For any given $\tau_{h} \in \Sigma_{h}$, there exist a function $v_{h} \in V_{h}$ such that

$$
\left(\tau_{h}, \varepsilon_{h}\left(v_{h}\right)\right)=\sum_{E \in \mathcal{E}_{h}^{\text {int }}} h_{E}\left\|\left[\left[\tau_{h} n\right]\right]\right\|_{E}^{2}
$$

and a positive constant $C_{2}$ such that

$$
\left\|v_{h}\right\|_{1, h} \leq C_{2}\left(\sum_{E \in \mathcal{E}_{h}^{\text {int }}} h_{E}\left\|\left[\left[\tau_{h} n\right]\right]\right\|_{E}^{2}\right)^{\frac{1}{2}} .
$$


Proof. Take $v_{h}$ satisfying

$$
\int_{E} v_{h} \mathrm{~d} s=h_{E} \int_{E}\left[\left[\tau_{h} n\right]\right] \mathrm{d} s, \quad E \in \mathcal{E}_{h}^{\text {int }}
$$

Then we have

$$
\left(\tau_{h}, \varepsilon_{h}\left(v_{h}\right)\right)=\sum_{E \in \mathcal{E}_{h}^{\text {int }}} \int_{E}\left[\left[\tau_{h} n\right]\right] \cdot v_{h} \mathrm{~d} s=\sum_{E \in \mathcal{E}_{h}^{\text {int }}} h_{E}\left\|\left[\left[\tau_{h} n\right]\right]\right\|_{E}^{2}
$$

which is (4.4).

For (4.5), it is sufficient to use the standard scaling arguments [15].

Next we verify the $Z_{h}$-ellipticity. According to Lemma 4.1, for any given $\tau_{h} \in Z_{h}$, there exists a function $v_{h} \in V_{h}$

$$
\left(\tau_{h}, \varepsilon_{h}\left(v_{h}\right)\right)=\sum_{E \in \mathcal{E}_{h}^{\text {int }}} h_{E}\left\|\left[\left[\tau_{h} n\right]\right]\right\|_{E}^{2}=0,
$$

which implies that the normal jumps of $\tau_{h}$ over interior edges (faces) vanish. Thus the divergence of $\tau_{h}$ belongs to $L^{2}(\Omega)^{d}$ and $\operatorname{div} \tau_{h}=0$. According to (3.5), we obtain the $Z_{h^{-}}$ ellipticity.

To prove the weaker inf-sup condition, we apply the Korn's inequality on piecewise $H^{1}$ vector space (cf. [8]) to the space $V_{h}$ and obtain

$$
\left\|v_{h}\right\|_{1, h}^{2} \leq C\left(\left\|\varepsilon_{h}\left(v_{h}\right)\right\|^{2}+\sum_{E \in \mathcal{E}_{h}} h_{E}^{-1}\left\|\left[\left[v_{h}\right]\right]\right\|_{E}^{2}\right), \quad \forall v_{h} \in V_{h},
$$

since the functions in $V_{h}$ are piecewise linear on $\mathcal{T}_{h}$. For any given $v_{h} \in V_{h}$, we take $\tau_{h}=$ $\varepsilon_{h}\left(v_{h}\right)$. Since

$$
\int_{\Omega} \operatorname{tr} \tau_{h} \mathrm{~d} x=\int_{\Omega} \operatorname{tr} \varepsilon_{h}\left(v_{h}\right) \mathrm{d} x=\sum_{K \in \mathcal{T}_{h}} \int_{K} \operatorname{div} v_{h} \mathrm{~d} x=\sum_{E \in \mathcal{E}_{h}} \int_{E}\left[\left[v_{h} \cdot n\right]\right] \mathrm{d} s=0,
$$

then we have $\tau_{h} \in \Sigma_{h}$. Therefore, there exist two positive constants $\beta_{1}$ and $\beta_{2}$ such that

$$
\frac{\left(\tau_{h}, \varepsilon_{h}\left(v_{h}\right)\right)}{\left\|\tau_{h}\right\|}=\left\|\varepsilon_{h}\left(v_{h}\right)\right\| \geq \beta_{1}\left\|v_{h}\right\|_{1, h}-\beta_{2}\left(\sum_{E \in \mathcal{E}_{h}} h_{E}^{-1}\left\|\left[\left[v_{h}\right]\right]\right\|_{E}^{2}\right)^{\frac{1}{2}},
$$

which leads to (4.3).

Based on the weaker inf-sup condition (4.3), we propose a stabilized mixed finite element method: find $\left(\sigma_{h}, u_{h}\right) \in \Sigma_{h} \times V_{h}$ satisfying

$$
\mathcal{Q}\left(\left(\sigma_{h}, u_{h}\right),\left(\tau_{h}, v_{h}\right)\right)=\left(f, v_{h}\right), \quad \forall\left(\tau_{h}, v_{h}\right) \in \Sigma_{h} \times V_{h},
$$


where the bilinear form is defined by

$$
\mathcal{Q}\left(\left(\sigma_{h}, u_{h}\right),\left(\tau_{h}, v_{h}\right)\right)=\left(\mathcal{A} \sigma_{h}, \tau_{h}\right)-\left(\tau_{h}, \varepsilon_{h}\left(u_{h}\right)\right)+\left(\sigma_{h}, \varepsilon_{h}\left(v_{h}\right)\right)+\gamma \sum_{E \in \mathcal{E}_{h}} h_{E}^{-1} \int_{E}\left[\left[u_{h}\right]\right] \cdot\left[\left[v_{h}\right]\right] \mathrm{d} s,
$$

and $\gamma$ is a positive constant to be properly chosen. Since $V_{h} \nsubseteq H_{0}^{1}(\Omega)^{d}$, the mixed method (4.6) is nonconforming.

Remark 4.1. We observe that the stabilized formulation (4.6) is indeed a special case of mixed DG method proposed in [14], where the discrete spaces are fully discontinuous polynomial spaces.

We conclude this section by showing the existence and uniqueness of the solution to (4.6). To this end, we assume $f=0$, then it is sufficient to show that the system of homogeneous equations has only zero solution. By setting $\tau_{h}=\sigma_{h}$ and $v_{h}=u_{h}$, we have

$$
\mathcal{Q}\left(\left(\sigma_{h}, u_{h}\right),\left(\sigma_{h}, u_{h}\right)\right)=\left(\mathcal{A} \sigma_{h}, \sigma_{h}\right)+\gamma \sum_{E \in \mathcal{E}_{h}} h_{E}^{-1}\left\|\left[\left[u_{h}\right]\right]\right\|_{E}^{2}=0,
$$

which leads to

$$
\left(\mathcal{A} \sigma_{h}, \sigma_{h}\right)=0 \quad \text { and }\left.\quad\left[\left[u_{h}\right]\right]\right|_{E}=0, \quad \forall E \in \mathcal{E}_{h} .
$$

Due to the fact that $\left(\sigma_{h}, \varepsilon_{h}\left(v_{h}\right)\right)=0$ for all $v_{h} \in V_{h}$, i.e., $\sigma_{h} \in Z_{h}$, the $Z_{h}$-ellipticity implies $\sigma_{h}=0$. Thus, setting $v_{h}=0$ in (4.6) leads to

$$
\left(\tau_{h}, \varepsilon_{h}\left(u_{h}\right)\right)=0, \quad \forall \tau_{h} \in \Sigma_{h}
$$

which, together with the weaker inf-sup condition (4.3) and the fact that the jump of $u_{h}$ on each edge (face) vanishes, yields $u_{h}=0$.

\section{Stability}

In order to show the optimal convergence properties of the mixed method (4.6), we here derive a weaker inf-sup condition of the bilinear form $\mathcal{Q}$ with respect to the norm on $\Sigma_{h} \times V_{h}$. To this end, we first introduce a special subspace of $\Sigma_{h}$ and several auxiliary lemmas.

Let $V_{h}^{*}=V_{h} \cap H_{0}^{1}(\Omega)$ be the usual conforming linear finite element space with the approximation property [15]

$$
\left|v-I_{h}^{*} v\right|_{m, K} \leq C h_{K}^{1-m}|v|_{1, \widetilde{K}^{\prime}} \quad m=0,1, \quad \forall K \in \mathcal{T}_{h},
$$

where $v \in H_{0}^{1}(\Omega), I_{h}^{*}$ is the usual Clément interpolation operator and $\widetilde{K}$ denotes the union of elements that share at least one point with $K$. Then we define a subspace $Z_{h}^{*}$ of $\Sigma_{h}$ by

$$
Z_{h}^{*}=\left\{\tau_{h} \in \Sigma_{h} ;\left(\tau_{h}, \varepsilon\left(v_{h}^{*}\right)\right)=0, \forall v_{h}^{*} \in V_{h}^{*}\right\} .
$$

In addition, in the proofs of the following lemmas, we will frequently use the trace inequality [15]

$$
\|v\|_{E}^{2} \leq C\left(h_{E}^{-1}\|v\|_{K}^{2}+h_{E}|v|_{1, K}^{2}\right), \quad \forall E \subset \partial K, \quad \forall K \in \mathcal{T}_{h} .
$$


Lemma 5.1. There exists a positive constant $C_{1}$ such that

$$
\left\|\operatorname{div} \tau_{h}\right\|_{-1} \leq C_{1}\left(\sum_{E \in \mathcal{E}_{h}^{\text {int }}} h_{E}\left\|\left[\left[\tau_{h} n\right]\right]\right\|_{E}^{2}\right)^{\frac{1}{2}}, \quad \forall \tau_{h} \in Z_{h}^{*}
$$

Proof. For any given $\tau_{h} \in Z_{h}^{*}$, first we have

$$
\begin{aligned}
\left\langle\operatorname{div} \tau_{h}, v\right\rangle & =-\left(\tau_{h}, \varepsilon(v)\right)=-\left(\tau_{h}, \varepsilon\left(v-I_{h}^{*} v\right)\right) \\
& =-\sum_{E \in \mathcal{E}_{h}^{\text {int }}} \int_{E}\left[\left[\tau_{h} n\right]\right] \cdot\left(v-I_{h}^{*} v\right) \mathrm{d} s \\
& \leq \sum_{E \in \mathcal{E}_{h}^{\text {int }}}\left\|\left[\left[\tau_{h} n\right]\right]\right\|_{E}\left\|v-I_{h}^{*} v\right\|_{E}
\end{aligned}
$$

where $v \in H_{0}^{1}(\Omega)^{d}$ is arbitrary. Combining (5.1) and (5.2), it holds

$$
\left\|v-I_{h}^{*} v\right\|_{E} \leq C h_{E}^{1 / 2}|v|_{1, \widetilde{K}^{\prime}} \quad \forall E \subset \partial K, \quad \forall K \in \mathcal{T}_{h},
$$

which implies

$$
\left\langle\operatorname{div} \tau_{h}, v\right\rangle \leq C|v|_{1}\left(\sum_{E \in \mathcal{E}_{h}^{\text {int }}} h_{E}\left\|\left[\left[\tau_{h} n\right]\right]\right\|_{E}^{2}\right)^{\frac{1}{2}} .
$$

According to the definition of $\|\cdot\|_{-1}$, the proof is completed.

Lemma 5.2. There exists a positive constant $C_{3}$ such that

$$
\left(\sum_{E \in \mathcal{E}_{h}} h_{E}^{-1}\left\|\left[\left[v_{h}\right]\right]\right\|_{E}^{2}\right)^{\frac{1}{2}} \leq C_{3}\left\|v_{h}\right\|_{1, h}, \quad \forall v_{h} \in V_{h} .
$$

Proof. Let

$$
P_{E} v_{h}=\frac{1}{h_{E}} \int_{E} v_{h} \mathrm{~d} s, \quad E \in \mathcal{E}_{h} .
$$

The trace inequality (5.2) and Poincaré/ Friedrichs inequality [38] imply that

$$
\begin{aligned}
h_{E}^{-1}\left\|\left[\left[v_{h}\right]\right]\right\|_{E}^{2} & =h_{E}^{-1}\left\|\left[\left[v_{h}-P_{E} v_{h}\right]\right]\right\|_{E}^{2} \\
& \leq C h_{E}^{-1} \sum_{K \in \mathcal{T}_{E}}\left(h_{E}^{-1}\left\|v_{h}-P_{E} v_{h}\right\|_{K}^{2}+h_{E}\left|v_{h}\right|_{1, K}^{2}\right) \\
& \leq C \sum_{K \in \mathcal{T}_{E}}\left|v_{h}\right|_{1, K^{\prime}}^{2}
\end{aligned}
$$

where $\mathcal{T}_{E}$ is the set of the elements having $E$ as an edge (or face). Thus the inequality (5.4) holds. 
For convenience, we also show that the compliance tensor $\mathcal{A}$ is bounded in the $L^{2}$ norm.

Lemma 5.3. There holds

$$
\|\mathcal{A} \tau\| \leq \frac{1}{2 \mu}\|\tau\|, \quad \forall \tau \in \Sigma .
$$

Proof. It follows from the definition (2.1b) that

$$
\begin{aligned}
\|\mathcal{A} \tau\|^{2} & =\frac{1}{4 \mu^{2}}\left(\|\tau\|^{2}-\frac{2 \lambda}{d \lambda+2 \mu}\|\operatorname{tr} \tau\|^{2}+\frac{d \lambda^{2}}{(d \lambda+2 \mu)^{2}}\|\operatorname{tr} \tau\|^{2}\right) \\
& =\frac{1}{4 \mu^{2}}\left(\|\tau\|^{2}-\frac{\lambda(d \lambda+4 \mu)}{(d \lambda+2 \mu)^{2}}\|\operatorname{tr} \tau\|^{2}\right) \\
& \leq \frac{1}{4 \mu^{2}}\|\tau\|^{2},
\end{aligned}
$$

which leads to (5.5).

With the above preparations, we are ready to show the weaker inf-sup condition of $\mathcal{Q}$ on the subspace $Z_{h}^{*} \times V_{h}$.

Theorem 5.1. For any given $\left(\sigma_{h}, u_{h}\right) \in Z_{h}^{*} \times V_{h}$, there exists a positive constant $C_{\gamma}$ that is related to the parameter $\gamma$ such that

$$
\sup _{\left(\tau_{h}, v_{h}\right) \in \Sigma_{h} \times V_{h} \backslash\{0\}} \frac{\mathcal{Q}\left(\left(\sigma_{h}, u_{h}\right),\left(\tau_{h}, v_{h}\right)\right)}{\left\|\tau_{h}\right\|+\left\|v_{h}\right\|_{1, h}} \geq C_{\gamma}\left(\left\|\sigma_{h}\right\|+\left\|u_{h}\right\|_{1, h}\right) .
$$

Proof. First we have

$$
\mathcal{Q}\left(\left(\sigma_{h}, u_{h}\right),\left(\sigma_{h}, u_{h}\right)\right)=\left(\mathcal{A} \sigma_{h}, \sigma_{h}\right)+\gamma \sum_{E \in \mathcal{E}_{h}} h_{E}^{-1}\left\|\left[\left[u_{h}\right]\right]\right\|_{E}^{2} .
$$

Due to the weaker inf-sup condition (4.3), there exists a function $\rho_{h} \in \Sigma_{h}$ such that

$$
\left(\rho_{h}, \varepsilon_{h}\left(u_{h}\right)\right) \geq \beta_{1}\left\|u_{h}\right\|_{1, h}^{2}-\beta_{2}\left\|u_{h}\right\|_{1, h}\left(\sum_{E \in \mathcal{E}_{h}} h_{E}^{-1}\left\|\left[\left[u_{h}\right]\right]\right\|_{E}^{2}\right)^{\frac{1}{2}},
$$

and $\left\|\rho_{h}\right\|=\left\|u_{h}\right\|_{1, h}$. Then we take $\left(\tau_{h}, v_{h}\right)=\left(-\theta_{1} \rho_{h}, 0\right)$ and obtain

$$
\begin{aligned}
& \mathcal{Q}\left(\left(\sigma_{h}, u_{h}\right),\left(-\theta_{1} \rho_{h}, 0\right)\right) \\
= & -\theta_{1}\left(\mathcal{A} \sigma_{h}, \rho_{h}\right)+\theta_{1}\left(\rho_{h}, \varepsilon_{h}\left(u_{h}\right)\right) \\
\geq & -\theta_{1}\left(\mathcal{A} \sigma_{h}, \rho_{h}\right)+\theta_{1} \beta_{1}\left\|u_{h}\right\|_{1, h}^{2}-\theta_{1} \beta_{2}\left\|u_{h}\right\|_{1, h}\left(\sum_{E \in \mathcal{E}_{h}} h_{E}^{-1}\left\|\left[\left[u_{h}\right]\right]\right\|_{E}^{2}\right)^{\frac{1}{2}} \\
\geq & -\theta_{1}\left\|\mathcal{A} \sigma_{h}\right\|\left\|u_{h}\right\|_{1, h}+\theta_{1} \beta_{1}\left\|u_{h}\right\|_{1, h}^{2}-\theta_{1} \beta_{2}\left\|u_{h}\right\|_{1, h}\left(\sum_{E \in \mathcal{E}_{h}} h_{E}^{-1}\left\|\left[\left[u_{h}\right]\right]\right\|_{E}^{2}\right)^{\frac{1}{2}} .
\end{aligned}
$$


We use the following inequalities

$$
\begin{aligned}
& \left\|\mathcal{A} \sigma_{h}\right\|\left\|u_{h}\right\|_{1, h} \leq \frac{\beta_{1}}{4}\left\|u_{h}\right\|_{1, h}^{2}+\frac{1}{\beta_{1}}\left\|\mathcal{A} \sigma_{h}\right\|^{2}, \\
& \beta_{2}\left\|u_{h}\right\|_{1, h}\left(\sum_{E \in \mathcal{E}_{h}} h_{E}^{-1}\left\|\left[\left[u_{h}\right]\right]\right\|_{E}^{2}\right)^{\frac{1}{2}} \leq \frac{\beta_{1}}{4}\left\|u_{h}\right\|_{1, h}^{2}+\frac{\beta_{2}^{2}}{\beta_{1}} \sum_{E \in \mathcal{E}_{h}} h_{E}^{-1}\left\|\left[\left[u_{h}\right]\right]\right\|_{E}^{2},
\end{aligned}
$$

and Lemma 5.3 to obtain

$$
\mathcal{Q}\left(\left(\sigma_{h}, u_{h}\right),\left(-\theta_{1} \rho_{h}, 0\right)\right) \geq \frac{\theta_{1} \beta_{1}}{2}\left\|u_{h}\right\|_{1, h}^{2}-\frac{\theta_{1}}{4 \beta_{1} \mu^{2}}\left\|\sigma_{h}\right\|^{2}-\frac{\theta_{1} \beta_{2}^{2}}{\beta_{1}} \sum_{E \in \mathcal{E}_{h}} h_{E}^{-1}\left\|\left[\left[u_{h}\right]\right]\right\|_{E}^{2} .
$$

Due to Lemma 4.1, there exists a function $w_{h} \in V_{h}$ such that

$$
\left(\sigma_{h}, \varepsilon_{h}\left(w_{h}\right)\right)=\sum_{E \in \mathcal{E}_{h}^{\text {int }}} h_{E}\left\|\left[\left[\sigma_{h} n\right]\right]\right\|_{E}^{2}
$$

and

$$
\left\|w_{h}\right\|_{1, h} \leq C_{2}\left(\sum_{E \in \mathcal{E}_{h}^{\text {int }}} h_{E}\left\|\left[\left[\sigma_{h} n\right]\right]\right\|_{E}^{2}\right)^{\frac{1}{2}}
$$

Then we take $\left(\tau_{h}, v_{h}\right)=\left(0, \theta_{2} w_{h}\right)$ and obtain

$$
\begin{aligned}
& \mathcal{Q}\left(\left(\sigma_{h}, u_{h}\right),\left(0, \theta_{2} w_{h}\right)\right)=\theta_{2}\left(\sigma_{h}, \mathcal{E}_{h}\left(w_{h}\right)\right)+\theta_{2} \gamma \sum_{E \in \mathcal{E}_{h}} h_{E}^{-1} \int_{E}\left[\left[u_{h}\right]\right] \cdot\left[\left[w_{h}\right]\right] \mathrm{d} s \\
= & \theta_{2} \sum_{E \in \mathcal{E}_{h}^{\text {int }}} h_{E}\left\|\left[\left[\sigma_{h} n\right]\right]\right\|_{E}^{2}+\theta_{2} \gamma \sum_{E \in \mathcal{E}_{h}} h_{E}^{-1} \int_{E}\left[\left[u_{h}\right]\right] \cdot\left[\left[w_{h}\right]\right] \mathrm{d} s \\
\geq & \theta_{2} \sum_{E \in \mathcal{E}_{h}^{\text {int }}} h_{E}\left\|\left[\left[\sigma_{h} n\right]\right]\right\|_{E}^{2}-\theta_{2} \gamma\left(\sum_{E \in \mathcal{E}_{h}} h_{E}^{-1}\left\|\left[\left[u_{h}\right]\right]\right\|_{E}^{2}\right)^{\frac{1}{2}}\left(\sum_{E \in \mathcal{E}_{h}} h_{E}^{-1}\left\|\left[\left[w_{h}\right]\right]\right\|_{E}^{2}\right)^{\frac{1}{2}} \\
\geq & \theta_{2} \sum_{E \in \mathcal{E}_{h}^{\text {int }}} h_{E}\left\|\left[\left[\sigma_{h} n\right]\right]\right\|_{E}^{2}-\theta_{2} \gamma C_{3}\left(\sum_{E \in \mathcal{E}_{h}} h_{E}^{-1}\left\|\left[\left[u_{h}\right]\right]\right\|_{E}^{2}\right)^{\frac{1}{2}}\left\|w_{h}\right\|_{1, h} \\
\geq & \theta_{2} \sum_{E \in \mathcal{E}_{h}^{\text {int }}} h_{E}\left\|\left[\left[\sigma_{h} n\right]\right]\right\|_{E}^{2}-\theta_{2} \gamma C_{2} C_{3}\left(\sum_{E \in \mathcal{E}_{h}} h_{E}^{-1}\left\|\left[\left[u_{h}\right]\right]\right\|_{E}^{2}\right)^{\frac{1}{2}}\left(\sum_{E \in \mathcal{E}_{h}^{\text {int }}} h_{E}\left\|\left[\left[\sigma_{h} n\right]\right]\right\|_{E}^{2}\right)^{\frac{1}{2}},
\end{aligned}
$$

where we have applied Lemma 5.2 to the second last inequality. 
We use the following inequality

$$
\begin{aligned}
& \gamma C_{2} C_{3}\left(\sum_{E \in \mathcal{E}_{h}} h_{E}^{-1}\left\|\left[\left[u_{h}\right]\right]\right\|_{E}^{2}\right)^{\frac{1}{2}}\left(\sum_{E \in \mathcal{E}_{h}^{\text {int }}} h_{E}\left\|\left[\left[\sigma_{h} n\right]\right]\right\|_{E}^{2}\right)^{\frac{1}{2}} \\
\leq & \frac{\gamma^{2} C_{2}^{2} C_{3}^{2}}{2} \sum_{E \in \mathcal{E}_{h}} h_{E}^{-1}\left\|\left[\left[u_{h}\right]\right]\right\|_{E}^{2}+\frac{1}{2} \sum_{E \in \mathcal{E}_{h}^{\text {int }}} h_{E}\left\|\left[\left[\sigma_{h} n\right]\right]\right\|_{E}^{2}
\end{aligned}
$$

to obtain

$$
\mathcal{Q}\left(\left(\sigma_{h}, u_{h}\right),\left(0, \theta_{2} w_{h}\right)\right) \geq \frac{\theta_{2}}{2} \sum_{E \in \mathcal{E}_{h}^{\text {int }}} h_{E}\left\|\left[\left[\sigma_{h} n\right]\right]\right\|_{E}^{2}-\frac{\theta_{2} \gamma^{2} C_{2}^{2} C_{3}^{2}}{2} \sum_{E \in \mathcal{E}_{h}} h_{E}^{-1}\left\|\left[\left[u_{h}\right]\right]\right\|_{E}^{2} .
$$

Summing up (5.7-5.9), we take $\left(\tau_{h}, v_{h}\right)=\left(\sigma_{h}-\theta_{1} \rho_{h}, u_{h}+\theta_{2} w_{h}\right)$ and obtain

$$
\begin{aligned}
& \mathcal{Q}\left(\left(\sigma_{h}, u_{h}\right),\left(\sigma_{h}-\theta_{1} \rho_{h}, u_{h}+\theta_{2} w_{h}\right)\right) \\
& \geq\left(\mathcal{A} \sigma_{h}, \sigma_{h}\right)+\frac{\theta_{2}}{2} \sum_{E \in \mathcal{E}_{h}^{J}} h_{E}\left\|\left[\left[\sigma_{h} n\right]\right]\right\|_{E}^{2}-\frac{\theta_{1}}{4 \beta_{1} \mu^{2}}\left\|\sigma_{h}\right\|^{2} \\
& \quad+\left(\gamma-\frac{\theta_{1} \beta_{2}^{2}}{\beta_{1}}-\frac{\theta_{2} \gamma^{2} C_{2}^{2} C_{3}^{2}}{2}\right) \sum_{E \in \mathcal{E}_{h}} h_{E}^{-1}\left\|\left[\left[u_{h}\right]\right]\right\|_{E}^{2}+\frac{\theta_{1} \beta_{1}}{2}\left\|u_{h}\right\|_{1, h}^{2} .
\end{aligned}
$$

Setting $\theta_{2}=\frac{1}{2 \gamma C_{2}^{2} C_{3}^{2}}$, it yields

$$
\gamma-\frac{\theta_{1} \beta_{2}^{2}}{\beta_{1}}-\frac{\theta_{2} \gamma^{2} C_{2}^{2} C_{3}^{2}}{2}=\frac{3 \gamma}{4}-\frac{\theta_{1} \beta_{2}^{2}}{\beta_{1}}
$$

which implies that

$$
\begin{aligned}
& \mathcal{Q}\left(\left(\sigma_{h}, u_{h}\right),\left(\sigma_{h}-\theta_{1} \rho_{h}, u_{h}+\theta_{2} w_{h}\right)\right) \\
\geq & \left(\mathcal{A} \sigma_{h}, \sigma_{h}\right)+\frac{1}{4 \gamma C_{2}^{2} C_{3}^{2}} \sum_{E \in \mathcal{E}_{h}^{\text {int }}} h_{E}\left\|\left[\left[\sigma_{h} n\right]\right]\right\|_{E}^{2}-\frac{\theta_{1}}{4 \beta_{1} \mu^{2}}\left\|\sigma_{h}\right\|^{2} \\
& +\left(\frac{3 \gamma}{4}-\frac{\theta_{1} \beta_{2}^{2}}{\beta_{1}}\right) \sum_{E \in \mathcal{E}_{h}} h_{E}^{-1}\left\|\left[\left[u_{h}\right]\right]\right\|_{E}^{2}+\frac{\theta_{1} \beta_{1}}{2}\left\|u_{h}\right\|_{1, h}^{2} \\
\geq & \left(\mathcal{A} \sigma_{h}, \sigma_{h}\right)+\frac{1}{4 \gamma C_{1}^{2} C_{2}^{2} C_{3}^{2}}\left\|\operatorname{div} \sigma_{h}\right\|_{-1}^{2}-\frac{\theta_{1}}{4 \beta_{1} \mu^{2}}\left\|\sigma_{h}\right\|^{2}+\left(\frac{3 \gamma}{4}-\frac{\theta_{1} \beta_{2}^{2}}{\beta_{1}}\right) \sum_{E \in \mathcal{E}_{h}} h_{E}^{-1}\left\|\left[\left[u_{h}\right]\right]\right\|_{E}^{2} \\
& \quad+\frac{\theta_{1} \beta_{1}}{2}\left\|u_{h}\right\|_{1, h}^{2} \\
\geq & \left(\alpha \min \left\{1, \frac{1}{4 \gamma C_{1}^{2} C_{2}^{2} C_{3}^{2}}\right\}-\frac{\theta_{1}}{4 \beta_{1} \mu^{2}}\right)\left\|\sigma_{h}\right\|^{2}+\left(\frac{3 \gamma}{4}-\frac{\theta_{1} \beta_{2}^{2}}{\beta_{1}}\right) \sum_{E \in \mathcal{E}_{h}} h_{E}^{-1}\left\|\left[\left[u_{h}\right]\right]\right\|_{E}^{2}+\frac{\theta_{1} \beta_{1}}{2}\left\|u_{h}\right\|_{1, h}^{2}
\end{aligned}
$$


where we have used (3.5) and Lemma 5.1, since $\sigma_{h} \in Z_{h}^{*}$.

Setting

$$
\theta_{1}=\min \left\{2 \alpha \beta_{1} \mu^{2} \min \left\{1, \frac{1}{4 \gamma C_{1}^{2} C_{2}^{2} C_{3}^{2}}\right\}, \frac{\beta_{1} \gamma}{4 \beta_{2}^{2}}\right\}
$$

it holds that

$$
\begin{aligned}
& \alpha \min \left\{1, \frac{1}{4 \gamma C_{1}^{2} C_{2}^{2} C_{3}^{2}}\right\}-\frac{\theta_{1}}{4 \beta_{1} \mu^{2}} \geq \frac{\alpha}{2} \min \left\{1, \frac{1}{4 \gamma C_{1}^{2} C_{2}^{2} C_{3}^{2}}\right\}, \\
& \frac{3 \gamma}{4}-\frac{\theta_{1} \beta_{2}^{2}}{\beta_{1}} \geq \frac{\gamma}{2}
\end{aligned}
$$

which leads to

$$
\begin{aligned}
& \mathcal{Q}\left(\left(\sigma_{h}, u_{h}\right),\left(\sigma_{h}-\theta_{1} \rho_{h}, u_{h}+\theta_{2} w_{h}\right)\right) \\
\geq & \frac{\alpha}{2} \min \left\{1, \frac{1}{4 \gamma C_{1}^{2} C_{2}^{2} C_{3}^{2}}\right\}\left\|\sigma_{h}\right\|^{2}+\frac{\gamma}{2} \sum_{E \in \mathcal{E}_{h}} h_{E}^{-1}\left\|\left[\left[u_{h}\right]\right]\right\|_{E}^{2}+\frac{\theta_{1} \beta_{1}}{2}\left\|u_{h}\right\|_{1, h}^{2} \\
\geq & C_{\gamma}\left(\left\|\sigma_{h}\right\|^{2}+\left\|u_{h}\right\|_{1, h}^{2}\right) .
\end{aligned}
$$

On the other hand, we have

$$
\begin{aligned}
& \left\|\sigma_{h}-\theta_{1} \rho_{h}\right\| \leq\left\|\sigma_{h}\right\|+\theta_{1}\left\|u_{h}\right\|_{1, h}, \\
& \left\|u_{h}+\theta_{2} w_{h}\right\|_{1, h} \leq\left\|u_{h}\right\|_{1, h}+C_{2} \theta_{2}\left(\sum_{E \in \mathcal{E}_{h}^{\text {int }}} h_{E}\left\|\left[\left[\sigma_{h} n\right]\right]\right\|_{E}^{2}\right)^{\frac{1}{2}} \leq C\left(\left\|\sigma_{h}\right\|+\left\|u_{h}\right\|_{1, h}\right),
\end{aligned}
$$

where we have used the trace inequality (5.2) and inverse inequality on finite element spaces.

Summing up (5.10-5.11b), the proof is completed.

Remark 5.1. We obseve that the weaker inf-sup condition of the bilinear form $\mathcal{Q}$ only holds on the subspace $Z_{h}^{*} \times V_{h}$. It is sufficient to derive the optimal convergence results of the mixed method.

\section{Error analysis}

This section is devoted to the error estimation on $\left\|\sigma-\sigma_{h}\right\|,\left\|u-u_{h}\right\|_{1, h}$ and $\left\|u-u_{h}\right\|$, where $(\sigma, u)$ is the weak solution to $(2.2)$ and $\left(\sigma_{h}, u_{h}\right)$ the approximate solution satisfying (4.6).

Theorem 6.1. Under the condition of the regularity estimate (2.3), it holds that

$$
\left\|\sigma-\sigma_{h}\right\|+\left\|u-u_{h}\right\|_{1, h} \leq C h\|f\| .
$$


Proof. Observing (2.2) and (4.6), we first reformulate the consistency error, for any $\left(\tau_{h}, v_{h}\right) \in$ $\Sigma_{h} \times V_{h}$,

$$
\begin{aligned}
& \mathcal{Q}\left(\left(\sigma-\sigma_{h}, u-u_{h}\right),\left(\tau_{h}, v_{h}\right)\right) \\
= & \mathcal{Q}\left((\sigma, u),\left(\tau_{h}, v_{h}\right)\right)-\left(f, v_{h}\right)=\left(\sigma, \varepsilon_{h}\left(v_{h}\right)\right)-\left(f, v_{h}\right) \\
= & \sum_{E \in \mathcal{E}_{h}} \int_{E} \sigma n \cdot\left[\left[v_{h}\right]\right] \mathrm{d} s=\sum_{E \in \mathcal{E}_{h}} \int_{E}\left(\sigma-P_{E} \sigma\right) n \cdot\left[\left[v_{h}-P_{E} v_{h}\right]\right] \mathrm{d} s,
\end{aligned}
$$

since the jump average of $v_{h}$ on each edge (face) vanishes. The Poincaré/Friedrichs inequality [38] and trace inequality (5.2) imply that

$$
\left\|\sigma-P_{E} \sigma\right\|_{E} \leq C h_{K}^{1 / 2}|\sigma|_{1, K}, \quad\left\|v_{h}-P_{E} v_{h}\right\|_{E} \leq C h_{K}^{1 / 2}\left|v_{h}\right|_{1, K}, \quad \forall E \subset \partial K, \quad \forall K \in \mathcal{T}_{h},
$$

which, together with (6.1), yields

$$
\mathcal{Q}\left(\left(\sigma-\sigma_{h}, u-u_{h}\right),\left(\tau_{h}, v_{h}\right)\right) \leq C h|\sigma|_{1}\left\|v_{h}\right\|_{1, h} .
$$

Observing the third equation of (6.1), there holds

$$
\left(\Pi_{h} \sigma-\sigma_{h}, \varepsilon\left(v_{h}\right)\right)=\left(\sigma-\sigma_{h}, \varepsilon\left(v_{h}\right)\right)=0, \quad \forall v_{h} \in V_{h}^{*},
$$

i.e., $\Pi_{h} \sigma-\sigma_{h} \in Z_{h}^{*}$. Then the weaker inf-sup condition (5.6) on $Z_{h}^{*} \times V_{h}$ leads to

$$
\begin{aligned}
& \left\|\sigma_{h}-\Pi_{h} \sigma\right\|+\left\|u_{h}-I_{h} u\right\|_{1, h} \leq \frac{1}{C_{\gamma}} \sup _{\left(\tau_{h}, v_{h}\right) \in \Sigma_{h} \times V_{h} \backslash\{0\}} \frac{\mathcal{Q}\left(\left(\sigma_{h}-\Pi_{h} \sigma, u_{h}-I_{h} u\right),\left(\tau_{h}, v_{h}\right)\right)}{\left\|\tau_{h}\right\|+\left\|v_{h}\right\|_{1, h}} \\
= & \frac{1}{C_{\gamma}} \sup _{\left(\tau_{h}, v_{h}\right) \in \Sigma_{h} \times V_{h} \backslash\{0\}} \frac{\mathcal{Q}\left(\left(\sigma-\Pi_{h} \sigma, u-I_{h} u\right),\left(\tau_{h}, v_{h}\right)\right)-\mathcal{Q}\left(\left(\sigma-\sigma_{h}, u-u_{h}\right),\left(\tau_{h}, v_{h}\right)\right)}{\left\|\tau_{h}\right\|+\left\|v_{h}\right\|_{1, h}} .
\end{aligned}
$$

By using the interpolation error estimates (4.1a-4.1b), trace inequality (5.2) and Lemma 5.2 , we obtain

$$
\mathcal{Q}\left(\left(\sigma-\Pi_{h} \sigma, u-I_{h} u\right),\left(\tau_{h}, v_{h}\right)\right) \leq C h\left(|\sigma|_{1}+|u|_{2}\right)\left(\left\|\tau_{h}\right\|+\left\|v_{h}\right\|_{1, h}\right) .
$$

Substituting (6.2) and (6.4) into (6.3) yields

$$
\left\|\sigma_{h}-\Pi_{h} \sigma\right\|+\left\|u_{h}-I_{h} u\right\|_{1, h} \leq C h\left(|\sigma|_{1}+|u|_{2}\right) .
$$

The triangle inequality and regularity estimate (2.3) conclude the proof.

Theorem 6.2. Under the condition of the regularity estimate (2.3), it holds that

$$
\left\|u-u_{h}\right\| \leq C h^{2}\|f\| .
$$


Proof. The proof relies on the usual duality argument. Let $\left(\sigma^{*}, u^{*}\right)$ be the solution to the auxiliary problem

$$
\begin{cases}\mathcal{A} \sigma^{*}-\varepsilon\left(u^{*}\right)=0 & \text { in } \Omega \\ -\operatorname{div} \sigma^{*}=u-u_{h} & \text { in } \Omega \\ u^{*}=0 & \text { on } \partial \Omega .\end{cases}
$$

From the regularity estimate (2.3), it immediately follows that

$$
\left\|\sigma^{*}\right\|_{1}+\left\|u^{*}\right\|_{2} \leq C\left\|u-u_{h}\right\|
$$

On both sides of the second equation of (6.5), we take an $L^{2}$-inner product with respect to $u-u_{h}$ and obtain

$$
\begin{array}{r}
\left\|u-u_{h}\right\|^{2}=-\left(\operatorname{div} \sigma^{*}, u-u_{h}\right)=\left(\sigma^{*}, \varepsilon_{h}\left(u-u_{h}\right)\right)+\sum_{E \in \mathcal{E}_{h}} \int_{E} \sigma^{*} n \cdot\left[\left[u_{h}\right]\right] \mathrm{d} s \\
=\left(\sigma^{*}-\Pi_{h} \sigma^{*}, \varepsilon_{h}\left(u-u_{h}\right)\right)+\left(\Pi_{h} \sigma^{*}, \varepsilon_{h}\left(u-u_{h}\right)\right) \\
\quad+\sum_{E \in \mathcal{E}_{h}} \int_{E}\left(\sigma^{*}-P_{E} \sigma^{*}\right) n \cdot\left[\left[\left(u_{h}-u\right)-P_{E}\left(u_{h}-u\right)\right]\right] \mathrm{d} s .
\end{array}
$$

For the second term above, the Eqs. (2.2), (4.6) and (6.5) imply that

$$
\begin{aligned}
\left(\Pi_{h} \sigma^{*}, \varepsilon_{h}\left(u-u_{h}\right)\right) & =\left(\mathcal{A}\left(\sigma-\sigma_{h}\right), \Pi_{h} \sigma^{*}-\sigma^{*}\right)+\left(\mathcal{A}\left(\sigma-\sigma_{h}\right), \sigma^{*}\right) \\
& =\left(\mathcal{A}\left(\sigma-\sigma_{h}\right), \Pi_{h} \sigma^{*}-\sigma^{*}\right)+\left(\sigma-\sigma_{h}, \varepsilon\left(u^{*}\right)\right) \\
& =\left(\mathcal{A}\left(\sigma-\sigma_{h}\right), \Pi_{h} \sigma^{*}-\sigma^{*}\right)+\left(\sigma-\sigma_{h}, \varepsilon\left(u^{*}-I_{h}^{*} u^{*}\right)\right),
\end{aligned}
$$

where in the last equation we have used the fact that $\left(\sigma-\sigma_{h}, \varepsilon\left(v_{h}\right)\right)=0$ for all $v_{h} \in V_{h}^{*}$. Combining (6.7) and (6.8), it yields

$$
\begin{gathered}
\left\|u-u_{h}\right\|^{2}=\left(\sigma^{*}-\Pi_{h} \sigma^{*}, \varepsilon_{h}\left(u-u_{h}\right)\right)+\left(\mathcal{A}\left(\sigma-\sigma_{h}\right), \Pi_{h} \sigma^{*}-\sigma^{*}\right)+\left(\sigma-\sigma_{h}, \varepsilon\left(u^{*}-I_{h}^{*} u^{*}\right)\right) \\
+\sum_{E \in \mathcal{E}_{h}} \int_{E}\left(\sigma^{*}-P_{E} \sigma^{*}\right) n \cdot\left[\left[\left(u_{h}-u\right)-P_{E}\left(u_{h}-u\right)\right]\right] \mathrm{d} s .
\end{gathered}
$$

By using the interpolation error estimates (4.1a)-(4.1b) and (5.1), Poincaré/ Fridrichs inequality [38] and trace inequality (5.2), we obtain

$$
\left\|u-u_{h}\right\|^{2} \leq C h\left(\left\|\sigma-\sigma_{h}\right\|+\left\|u-u_{h}\right\|_{1, h}\right)\left(\left|\sigma^{*}\right|_{1}+\left|u^{*}\right|_{2}\right) .
$$

Combining the regularity estimate (6.6) and Theorem 6.1 concludes the proof. 


\section{Relation to a stabilized nonconforming pure displacement method}

As is stated in Introduction, for finite $\lambda$ the mixed method (4.6) can be reduced to the stabilized pure displacement method: find $u_{h} \in V_{h}$ satisfying

$$
\left(\mathcal{C} \varepsilon_{h}\left(u_{h}\right), \varepsilon_{h}\left(v_{h}\right)\right)+\gamma \sum_{E \in \mathcal{E}_{h}} h_{E}^{-1} \int_{E}\left[\left[u_{h}\right]\right] \cdot\left[\left[v_{h}\right]\right] \mathrm{d} s=\left(f, v_{h}\right), \quad \forall v_{h} \in V_{h}
$$

with $\sigma_{h}=\mathcal{C} \varepsilon_{h}\left(u_{h}\right)$, since $\varepsilon_{h}\left(V_{h}\right) \subset \Sigma_{h}$.

The stabilized nonconforming method has been proposed by Hansbo and Larson in [21]. Therein, the optimal convergence order has also been obtained uniformly in the nearly incompressible case. However, the error analysis was carried out in a mesh dependent energy-like norm defined as

$$
\|\| v \|_{h}=\left(\left(\mathcal{C} \varepsilon_{h}(v), \varepsilon_{h}(v)\right)+\sum_{E \in \mathcal{E}_{h}} h_{E} \int_{E}\left\{\left\{\mathcal{C} \varepsilon_{h}(v) n\right\}\right\} \cdot\left\{\left\{\varepsilon_{h}(v) n\right\}\right\} \mathrm{d} s+2 \mu \sum_{E \in \mathcal{E}_{h}} h_{E}^{-1}\|[[v]]\|_{E}^{2}\right),
$$

where $\{\{\cdot\}\}$ denotes the average operator on edges (or faces).

By contrast, we use the $L^{2}$-norm for the stress space and broken $H^{1}$-norm for the displacement space, which are more natural and completely independent of the mesh size and the Lamé constant. Moreover, as well as the displacement, the optimal convergence order for the stress tensor is also obtained uniformly in the nearly incompressible or incompressible case.

\section{Numerical results}

In this section, we demonstrate the optimal convergence and stability of the stabilized nonconforming mixed method by an example taken from [26]. To this end, we use the mixed formulation (4.6) with $\gamma=1$ to simultaneously approximate the stress and the displacement, although for finite $\lambda$ it is equivalent to the stabilized pure displacement method (7.1) that is easier to compute. Note that, the stabilized nonconforming method (7.1) has been tested to be locking-free by solving a closed cavity flow problem in [21]. Here, the numerical results are slightly better than those in [26].

In the following example, let $\Omega=(-1,1) \times(-1,1)$ and $\mu=1$. As shown in Fig. 1, we use the uniform $n \times n$ mesh $\mathcal{T}_{h}$ on $\Omega$ with $h=2 / n$. The right-side term is taken as

$$
f\left(x_{1}, x_{2}\right)=\left(\begin{array}{l}
-8\left(x_{1}+x_{2}\right)\left(\left(3 x_{1} x_{2}-2\right)\left(x_{1}^{2}+x_{2}^{2}\right)+5\left(x_{1} x_{2}-1\right)^{2}-2 x_{1}^{2} x_{2}^{2}\right) \\
-8\left(x_{1}-x_{2}\right)\left(\left(3 x_{1} x_{2}+2\right)\left(x_{1}^{2}+x_{2}^{2}\right)-5\left(x_{1} x_{2}+1\right)^{2}+2 x_{1}^{2} x_{2}^{2}\right)
\end{array}\right) .
$$

It is not difficult to check the exact solution of (2.2) in this case is

$$
u\left(x_{1}, x_{2}\right)=\left(\begin{array}{r}
-4 x_{2}\left(1-x_{2}^{2}\right)\left(1-x_{1}^{2}\right)^{2} \\
4 x_{1}\left(1-x_{1}^{2}\right)\left(1-x_{2}^{2}\right)^{2}
\end{array}\right)+\frac{1}{2+\lambda}\left(\begin{array}{c}
-4 x_{1}\left(1-x_{1}^{2}\right)\left(1-x_{2}^{2}\right)^{2} \\
-4 x_{2}\left(1-x_{2}^{2}\right)\left(1-x_{1}^{2}\right)^{2}
\end{array}\right),
$$




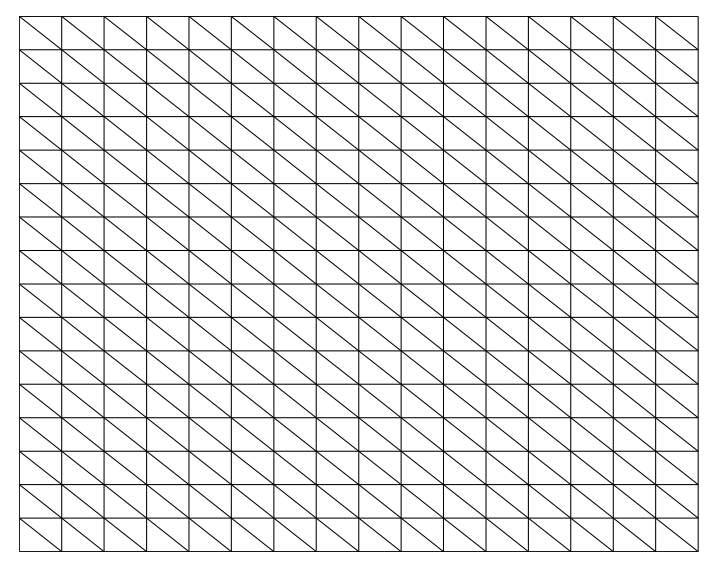

Figure 1: The uniform mesh with $h=2^{-3}$.

and the corresponding stress tensor $\sigma$ computed by $\sigma=\mathcal{C} \varepsilon(u)$.

We first discuss the convergence of our mixed method. The Lamé constant $\lambda$ is taken to be 1,10 and $10^{9}$, respectively. The corresponding numerical results for different values of $\lambda$ are given in Tables 1-3 and Figs. 2-4, from which we may find that the convergence order of $\left\|u-u_{h}\right\|,\left\|u-u_{h}\right\|_{1, h}$ and $\left\|\sigma-\sigma_{h}\right\|$ is $\mathcal{O}\left(h^{2}\right), \mathcal{O}(h)$ and $\mathcal{O}(h)$, respectively. It is

Table 1: The errors for $\lambda=1$ and different $h$.

\begin{tabular}{||cccc||}
$h$ & $\left\|u-u_{h}\right\|$ & $\left\|u-u_{h}\right\|_{1, h}$ & $\left\|\sigma-\sigma_{h}\right\|$ \\
$2^{0}$ & $1.2513 \mathrm{e}+000$ & $7.2810 \mathrm{e}+000$ & $1.1581 \mathrm{e}+001$ \\
$2^{-1}$ & $4.1688 \mathrm{e}-001$ & $4.2304 \mathrm{e}+000$ & $6.6350 \mathrm{e}+000$ \\
$2^{-2}$ & $1.0501 \mathrm{e}-001$ & $2.1569 \mathrm{e}+000$ & $3.4760 \mathrm{e}+000$ \\
$2^{-3}$ & $2.6279 \mathrm{e}-002$ & $1.0744 \mathrm{e}+000$ & $1.7484 \mathrm{e}+000$ \\
$2^{-4}$ & $6.5728 \mathrm{e}-003$ & $5.3408 \mathrm{e}-001$ & $8.7353 \mathrm{e}-001$ \\
$2^{-5}$ & $1.6438 \mathrm{e}-003$ & $2.6599 \mathrm{e}-001$ & $4.3622 \mathrm{e}-001$ \\
$2^{-6}$ & $4.1103 \mathrm{e}-004$ & $1.3270 \mathrm{e}-001$ & $2.1793 \mathrm{e}-001$ \\
\hline
\end{tabular}

Table 2: The errors for $\lambda=10$ and different $h$.

\begin{tabular}{||cccc||}
\hline$h$ & $\left\|u-u_{h}\right\|$ & $\left\|u-u_{h}\right\|_{1, h}$ & $\left\|\sigma-\sigma_{h}\right\|$ \\
\hline $2^{0}$ & $1.3084 \mathrm{e}+000$ & $7.0572 \mathrm{e}+000$ & $1.3423 \mathrm{e}+001$ \\
$2^{-1}$ & $4.3872 \mathrm{e}-001$ & $4.1392 \mathrm{e}+000$ & $7.6216 \mathrm{e}+000$ \\
$2^{-2}$ & $1.1381 \mathrm{e}-001$ & $2.1187 \mathrm{e}+000$ & $3.9040 \mathrm{e}+000$ \\
$2^{-3}$ & $2.8797 \mathrm{e}-002$ & $1.0557 \mathrm{e}+000$ & $1.9441 \mathrm{e}+000$ \\
$2^{-4}$ & $7.2248 \mathrm{e}-003$ & $5.2481 \mathrm{e}-001$ & $9.6844 \mathrm{e}-001$ \\
$2^{-5}$ & $1.8084 \mathrm{e}-003$ & $2.6139 \mathrm{e}-001$ & $4.8325 \mathrm{e}-001$ \\
$2^{-6}$ & $4.5236 \mathrm{e}-004$ & $1.3041 \mathrm{e}-001$ & $2.4138 \mathrm{e}-001$ \\
\hline
\end{tabular}




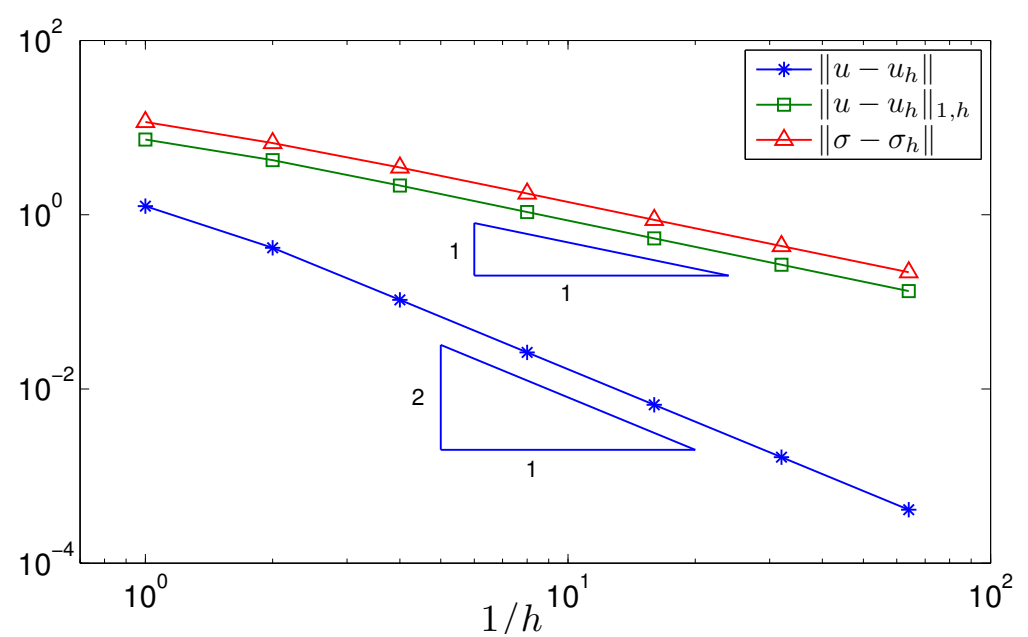

Figure 2: The errors for $\lambda=1$ and different $h$ in the logarithmic scale.

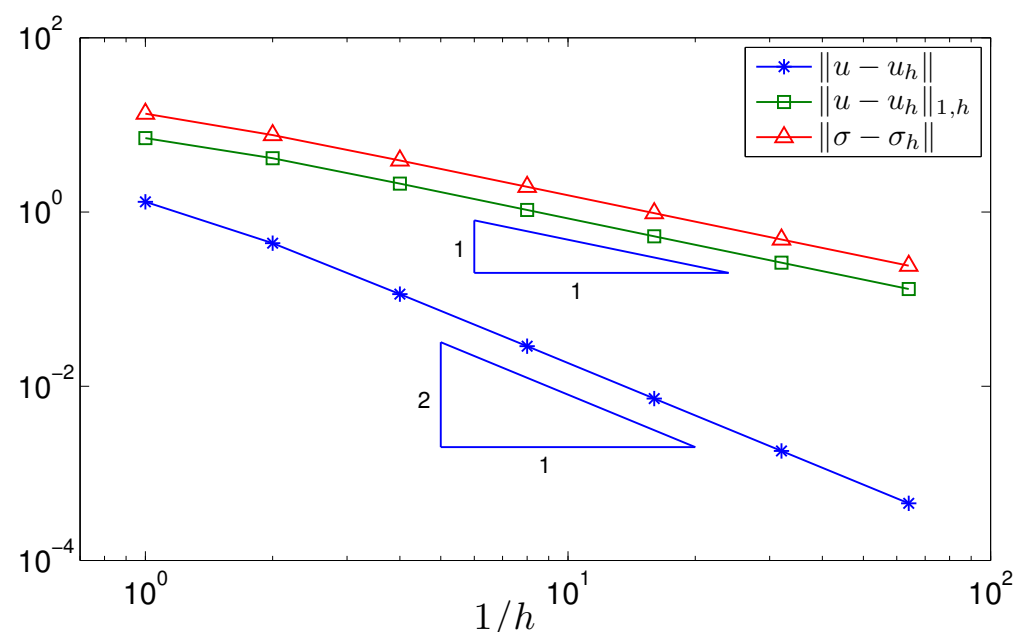

Figure 3: The errors for $\lambda=10$ and different $h$ in the logarithmic scale.

worth noticing that, even in the nearly incompressible case $\left(\lambda=10^{9}\right)$, the optimal convergence order can be still maintained. These numerical results are consistent with the theoretical results in Theorems 6.1 and 6.2.

Next, we focus on the robustness of the stabilized mixed method with respect to $\lambda$. Let $\lambda$ vary from 1 to $10^{9}$ on a fixed mesh with $h=2^{-6}$. The corresponding numerical results for $\left\|u-u_{h}\right\|,\left\|u-u_{h}\right\|_{1, h}$ and $\left\|\sigma-\sigma_{h}\right\|$ are shown in Table 4 , from which we observe that the errors are hardly affected by the choice of $\lambda$. Therefore, the stabilized nonconforming mixed method is stable with respect to $\lambda$, i.e., it is locking-free. 


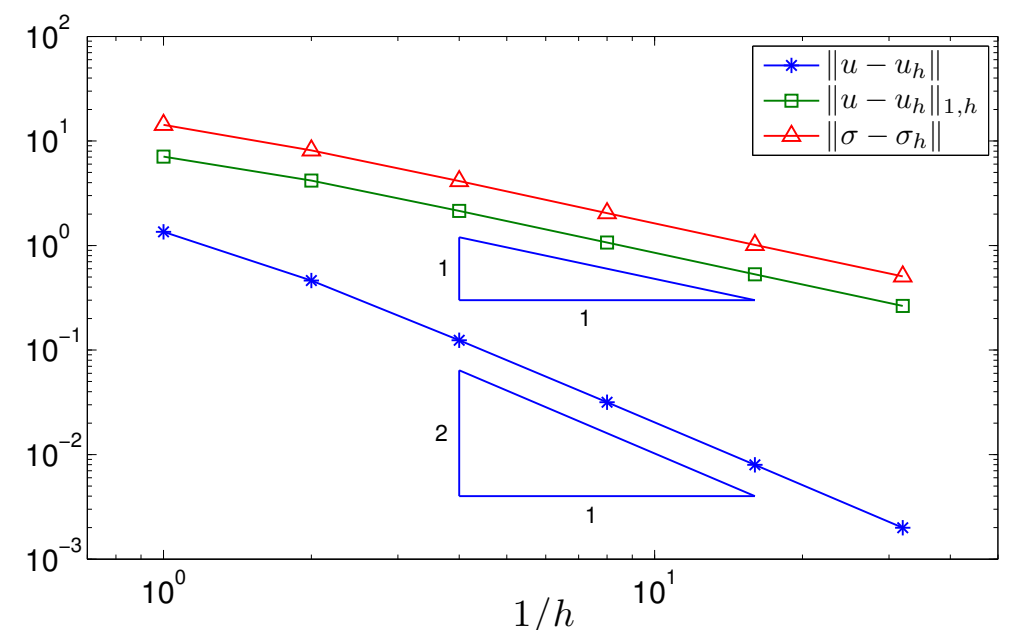

Figure 4: The errors for $\lambda=10^{9}$ and different $h$ in the logarithmic scale.

Table 3: The errors for $\lambda=10^{9}$ and different $h$.

\begin{tabular}{||cccc||}
\hline$h$ & $\left\|u-u_{h}\right\|$ & $\left\|u-u_{h}\right\|_{1, h}$ & $\left\|\sigma-\sigma_{h}\right\|$ \\
\hline $2^{0}$ & $1.3495 \mathrm{e}+000$ & $7.0870 \mathrm{e}+000$ & $1.4253 \mathrm{e}+001$ \\
$2^{-1}$ & $4.6211 \mathrm{e}-001$ & $4.1743 \mathrm{e}+000$ & $8.1347 \mathrm{e}+000$ \\
$2^{-2}$ & $1.2387 \mathrm{e}-001$ & $2.1422 \mathrm{e}+000$ & $4.1311 \mathrm{e}+000$ \\
$2^{-3}$ & $3.1715 \mathrm{e}-002$ & $1.0670 \mathrm{e}+000$ & $2.0420 \mathrm{e}+000$ \\
$2^{-4}$ & $7.9742 \mathrm{e}-003$ & $5.3016 \mathrm{e}-001$ & $1.0144 \mathrm{e}+000$ \\
$2^{-5}$ & $1.9958 \mathrm{e}-003$ & $2.6402 \mathrm{e}-001$ & $5.0577 \mathrm{e}-001$ \\
\hline
\end{tabular}

Table 4: The errors for $h=2^{-5}$ and different $\lambda$.

\begin{tabular}{||cccc||}
\hline$\lambda$ & $\left\|u-u_{h}\right\|$ & $\left\|u-u_{h}\right\|_{1, h}$ & $\left\|\sigma-\sigma_{h}\right\|$ \\
\hline $10^{0}$ & $1.6438 \mathrm{e}-003$ & $2.6599 \mathrm{e}-001$ & $4.3622 \mathrm{e}-001$ \\
$10^{1}$ & $1.8084 \mathrm{e}-003$ & $2.6139 \mathrm{e}-001$ & $4.8325 \mathrm{e}-001$ \\
$10^{2}$ & $1.9678 \mathrm{e}-003$ & $2.6360 \mathrm{e}-001$ & $5.0296 \mathrm{e}-001$ \\
$10^{3}$ & $1.9929 \mathrm{e}-003$ & $2.6398 \mathrm{e}-001$ & $5.0548 \mathrm{e}-001$ \\
$10^{4}$ & $1.9955 \mathrm{e}-003$ & $2.6401 \mathrm{e}-001$ & $5.0574 \mathrm{e}-001$ \\
$10^{5}$ & $1.9958 \mathrm{e}-003$ & $2.6402 \mathrm{e}-001$ & $5.0577 \mathrm{e}-001$ \\
$10^{6}$ & $1.9958 \mathrm{e}-003$ & $2.6402 \mathrm{e}-001$ & $5.0577 \mathrm{e}-001$ \\
$10^{7}$ & $1.9958 \mathrm{e}-003$ & $2.6402 \mathrm{e}-001$ & $5.0577 \mathrm{e}-001$ \\
$10^{8}$ & $1.9958 \mathrm{e}-003$ & $2.6402 \mathrm{e}-001$ & $5.0577 \mathrm{e}-001$ \\
$10^{9}$ & $1.9958 \mathrm{e}-003$ & $2.6402 \mathrm{e}-001$ & $5.0577 \mathrm{e}-001$ \\
\hline
\end{tabular}

\section{Conclusions}

In this paper, we present the stabilized mixed finite element method with the jump penalty term for the displacement, which is the discretization of the primal mixed varia- 
tional formulation for linear elasticity. Here we use the piecewise constant space for stress and the Crouzeix-Raviart element space for displacement, so the mixed method is nonconforming. The optimal convergence order is shown in the $L^{2}$-norm for stress and in the broken $H^{1}$-norm and $L^{2}$-norm for displacement, respectively. Moreover, the convergence does not deteriorate in the nearly incompressible or incompressible case, so the mixed method is locking-free. It is worth mentioning that, for finite $\lambda$ our mixed method can be reduced to the stabilized nonconforming method [21] based on the pure displacement formulation, where the error analysis was carried out in a mesh dependent energy-like norm as mentioned above. By contrast, the norms used in this paper are more natural and completely independent of the mesh size and the Lamé constant.

\section{Acknowledgements}

This work is supported by National Natural Science Foundation of China (No. 11701522), Key scientific research projects in colleges and universities in Henan Province (No. 18A110030) and Research Foundation for Advanced Talents of Henan University of Technology (No. 2018BS013).

\section{References}

[1] S. AdAmS AND B. COCKBURn, A mixed finite element method for elasticity in three dimensions, J. Sci. Comput., 25 (2005), pp. 515-521.

[2] D. N. ARNOlD, G. AWANOU AND R. WinTHER, Finite elements for symmetric tensors in three dimensions, Math. Comput., 77 (2008), pp. 1229-1251.

[3] D. N. ARnOlD, G. AWANOU AND R. Winther, Nonconforming tetrahedral mixed finite elements for elasticity, Math. Models Methods Appl. Sci., 24 (2014), pp. 783-796.

[4] D. N. ARnOld, F. BrezZI AND J. Douglas, JR., PEERS: a new mixed finite element for plane elasticity, Japan J. Appl. Math., 1 (1984), pp. 347-367.

[5] D. N. ARnold, J. Douglas, JR. AND C. P. GuPTA, A family of higher order mixed finite element methods for plane elasticity, Numer. Math., 45 (1984), pp. 1-22.

[6] D. N. ARnold AND R. Winther, Mixed finite elements for elasticity, Numer. Math., 92 (2002), pp. 401-419.

[7] D. N. ARNOld AND R. Winther, Nonconforming mixed elements for elasticity, Math. Models Methods Appl. Sci., 13 (2003), pp. 295-307.

[8] S. C. BRENNER, Korn's inequalities for piecewise $H^{1}$ vector fields, Math. Comput., 73 (2004), pp. 1067-1087.

[9] S. C. BRENNER AND L.-Y. SUNG, Linear finite element methods for planar linear elasticity, Math. Comput., 59 (1992), pp. 321-338.

[10] Z. CAI AND G. STARKE, First-order system least squares for the stress-displacement formulation: linear elasticity, SIAM J. Numer. Anal., 41 (2003), pp. 715-730.

[11] G. CHEN AND M. FENG, Stabilized finite element methods for Biot's consolidation problems using equal order elements, Adv. Appl. Math. Mech., 10 (2018), pp. 77-99.

[12] L. CHEN, J. HU AND X. HUANG, Stabilized mixed finite element methods for linear elasticity on simplicial grids in $\mathbb{R}^{n}$, Comput. Methods Appl. Math., 17 (2017), pp. 17-31. 
[13] S. CHEN, G. REN AND S. MAO, Second-order locking-free nonconforming elements for planar linear elasticity, J. Comput. Appl. Math., 233 (2010), pp. 2534-2548.

[14] Y. Chen, J. HuAnG, X. HuANG AND Y. XU, On the local discontinuous Galerkin method for linear elasticity, Math. Probl. Eng., (2010), article ID: 759547.

[15] P. G. Ciarlet, The Finite Element Method for Elliptic Problems, North Holland, Amsterdam, 1978.

[16] P. G. CiARlet, Mathematical Elasticity Volume I: Three-Dimensional Elasticity, NorthHolland, Amsterdam, 1988.

[17] M. CROUZEIX AND P. RAVIART, Conforming and nonconforming finite element methods for solving the stationary Stokes equations, RAIRO Modél. Math. Anal. Numér., 3 (1973), pp. 33-75.

[18] R. S. FALK, Nonconforming finite element methods for the equations of linear elasticity, Math. Comput., 57 (1991), pp. 529-550.

[19] L. P. Franca And T. J. Hughes, Two classes of mixed finite element methods, Comput. Method. Appl. M., 69 (1988), pp. 89-129.

[20] P. G. GRISVARD, Singularities in Boundary Value Problems, Springer, Berlin, 1992.

[21] P. HANSBO AND M. G. LARSON, Discontinuous Galerkin and the Crouzeix-Raviart element: application to elasticity, ESAIM-Math. Model. Numer. Anal., 37 (2003), pp. 63-72.

[22] J. HU, Finite element approximations of symmetric tensors on simplicial grids in $\mathbb{R}^{n}$ : the higher order case, J. Comput. Math., 33 (2015), pp. 283-296.

[23] J. HU AND Z.-C. SHI, Lower order rectangular nonconforming mixed finite elements for plane elasticity, SIAM J. Numer. Anal., 46 (2007/08), pp. 88-102.

[24] J. HU AND S. ZHANG, A family of conforming mixed finite elements for linear elasticity on triangular grids, arXiv preprint arXiv:1406.7457, (2014).

[25] J. HU AND S. ZHANG, A family of symmetric mixed finite elements for linear elasticity on tetrahedral grids, Sci. China Math., 58 (2015), pp. 297-307.

[26] X. HUANG AND J. HuANG, The compact discontinuous Galerkin method for nearly incompressible linear elasticity, J. Sci. Comput., 56 (2013), pp. 291-318.

[27] C. JOHNSON AND B. MERCIER, Some equilibrium finite element methods for two-dimensional elasticity problems, Numer. Math., 30 (1978), pp. 103-116.

[28] C. Johnson AND J. PItKÄRAnta, Analysis of some mixed finite element methods related to reduced integration, Math. Comput., 38 (1982), pp. 375-400.

[29] K. Y. KIM, Analysis of some low-order nonconforming mixed finite elements for linear elasticity problem, Numer. Meth. Partial Differential Equations, 22 (2006), pp. 638-660.

[30] C.-O. LEE, J. LEE AND D. SHEEN, A locking-free nonconforming finite element method for planar linear elasticity, Adv. Comput. Math., 19 (2003), pp. 277-291.

[31] S. MAO AND S. CHEN, A quadrilateral nonconforming finite element for linear elasticity problem, Adv. Comput. Math., 28 (2008), pp. 81-100.

[32] M. E. MORLEY, A family of mixed finite elements for linear elasticity, Numer. Math., 55 (1989), pp. 633-666.

[33] S. NiCAISE, Regularity of the solutions of elliptic systems in polyhedral domains, Belg. Math. Soc. Simon Stevin, 4 (1997), pp. 411-429.

[34] I. Soleimani, Y. T. Beni And F. Mehralian, A new size-dependent cylindrical shell element based on modified couple stress theory, Adv. Appl. Math. Mech., 10 (2018), pp. 819-844.

[35] R. STENBERG, A family of mixed finite elements for the elasticity problem, Numer. Math., 53 (1988), pp. 513-538.

[36] R. STENBERG AND M. SURI, Mixed hp finite element methods for problems in elasticity and Stokes flow, Numer. Math., 72 (1996), pp. 367-389. 
[37] M. VogeliUs, An analysis of the p-version of the finite element method for nearly incompressible materials. Uniformly valid, optimal error estimates, Numer. Math., 41 (1983), pp. 39-53.

[38] M. VOHRALÍK, On the discrete Poincaré-Friedrichs inequalities for nonconforming approximations of the Sobolev space $H^{1}$, Numer. Func. Anal. Opt., 26 (2005), pp. 925-952.

[39] Y. YANG AND S. ZHANG, Mixed finite element methods for elastodynamics problems in the symmetric formulation, Adv. Appl. Math. Mech., 10 (2018), pp. 1279-1304.

[40] Z. ZHANG, Analysis of some quadrilateral nonconforming elements for incompressible elasticity, SIAM J. Numer. Anal., 34 (1997), pp. 640-663. 